\title{
Laser Induced Single Events in SRAMs
}

\author{
C. Palomar ${ }^{1}$, I. López-Calle ${ }^{1,2}$, F. J Franco ${ }^{1}$, J. G. Izquierdo ${ }^{3}$, and J. A. Agapito ${ }^{1}$ \\ ${ }^{1}$ Dep. Física Aplicada III, Facultad de Físicas, Universidad Complutense de Madrid (UCM), 28040 Madrid (Spain) \\ (email: carlos.palomar, fjfranco, agapito@ fis.ucm.es). \\ ${ }^{2}$ European Space Agency, ESA/ESTEC-TEC/QEC, 2200 AG, Noorwijk (The Netherlands) (email: isabel.lopez- \\ calle@esa.int). \\ ${ }^{3}$ Centro de Láseres Ultrarrápidos (CLUR), Facultad de Químicas, Universidad Complutense de Madrid (UCM), 28040 \\ Madrid (Spain) (email: jegonzal@quim.ucm.es).
}

\begin{abstract}
Abstact - This paper is aimed to emulating the errors in semiconductor memories for atmospheric radiation by pulsed laser that acts as an ion. A sensitivity map of the memory is performed identifying potential error areas and how many errors occur simultaneously.
\end{abstract}

Keywords - Laser, memory, errors, SEU, MCU, sensitivity map.

\section{INTRODUCTION}

Today Single Event Effect (SEE) characterization of electronic components is carried out at heavy ion and proton facilities. In recent years, SEE laser test systems have become available to deviate irradiation tests performed at particle accelerators. Laser SEE tests are useful for component screening purposes and also in conjunction with EEE component hardening efforts (e.g. by detection of sensitive nodes). It has been demonstrated that the pulsed laser technique can reproduce SEEs in most EEE components [1 - 3]. The test method is also less costly than irradiation tests carried out at particle accelerators. This paper is aimed to emulating the errors in semiconductor memories for atmospheric radiation by pulsed laser that acts as an ion. A sensitivity map of the memory is performed identifying potential error areas and how many errors occur simultaneously.

\section{LASER FACILITY}

The experiments were performed at the UCM Ultrafast Lasers Center. The system is based on a femtosecond pulsed laser source with pulse rate of 1 $\mathrm{kHz}$. The laser system is flexible with the following tunable parameters for laser irradiation tests: wavelength, spot size, pulse energy, single shot, and multiple shot. Typically for SEE laser test facilities, the pulse width and wavelength are fixed parameters but, at the UCM-Spain, the wavelength is variable and can be chosen from ultraviolet $(300 \mathrm{~nm})$ to infrared $(3000$ $\mathrm{nm}$ ) while keeping the same pulse width (60 fs). In this case, the laser wavelength was set to $800 \mathrm{~nm}$ in order to create free charge in the surface of the device. A more complete description of the laser can be found in [1].

\section{EXPERIMENTAL SET UP}

The laser radiation process requires very delicate steps that are summarized below:

-Decapsulation and memory sizing. The laser radiation requires direct lighting device, so that previously we must perform its decapsulation. Decapsulation techniques depend on the package or integrated circuit packaging and you must choose the right solution. Since the selected memories are plastic encapsulation, the more effective technique for decapsulation packaged plastic polymer is by etching. For the type of polymer typically used in commercial encapsulated the suitable acid is nitric red acid at a temperature of about $75-80^{\circ} \mathrm{C}$. Because this acid attacks the polymer and the metal, the decapsulation by the top of the chip is much more destructive because, the acid also attacks the metal contacts, pins and even integrated circuit tracks. The device decapsulation is also necessary to study the "lay-out" or integrated circuit design and XYZ sized necessary to define the conditions of illumination and laser scanning. The lay-out and memory size of the memories AS6C6264 and CY62256 are shown in Fig.1 and Fig. 2 respectively.

-Build test boards. After decapsulation and sized integrated circuit, it is necessary to design a printed circuit board for electronic configuration and polarization levels. Figure 3 shows the printed circuit board that has been designed and manufactured for control of the memory and communication with a computer for data collection. 


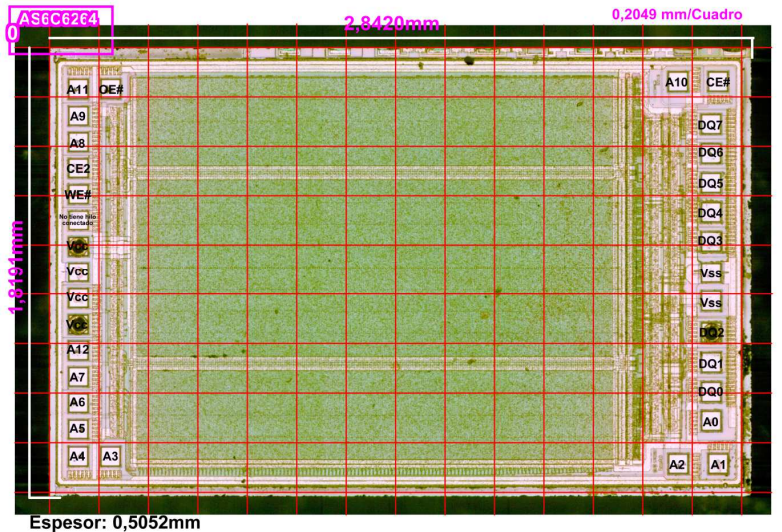

Fig.1. Lay-out and size of the memory AS6C6264 (Alliance).

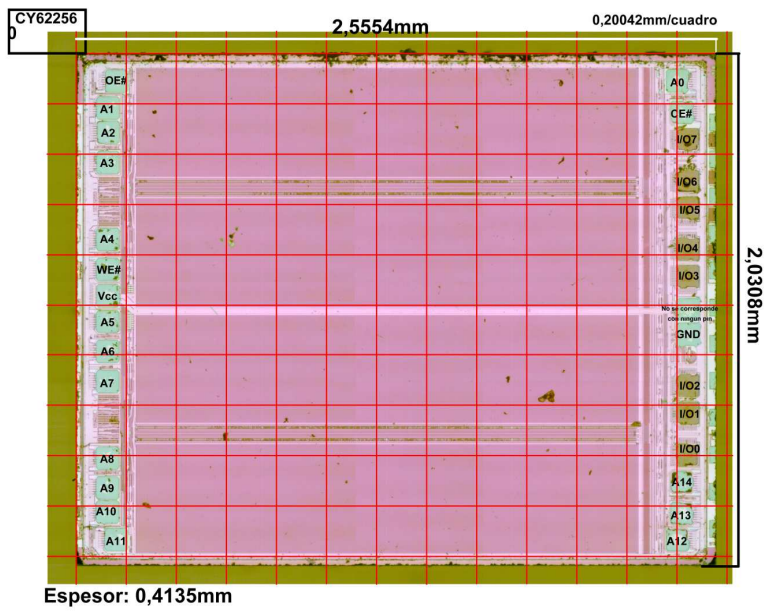

Fig.2. Lay-out and size of the memory AS6C6264 (Alliance).

The microprocessor (right) communicates with a PC through a USB port (via RS232 serial converter wire to USB). On the left, in a separate board which will be subject to the sample holder or platform for the XYZ movement front of the laser beam is positioned to radiate memory, which communicates with the microprocessor through an address bus and data.

Once built the circuit said microprocessor is programmed to:

- Write the memory with the desired pattern. The program offers the choice between a large number of patterns, such as: all zeros or all ones, or turn or random ones and zeros.

- $\quad$ Read the contents of memory.

- Check the status of all memory cells, ie read all memory locations, compared with the pattern written in a file and store the result of the comparison.

- Transmit to PC memory status. The errors, if any, and the address of the cells in which they were produced.

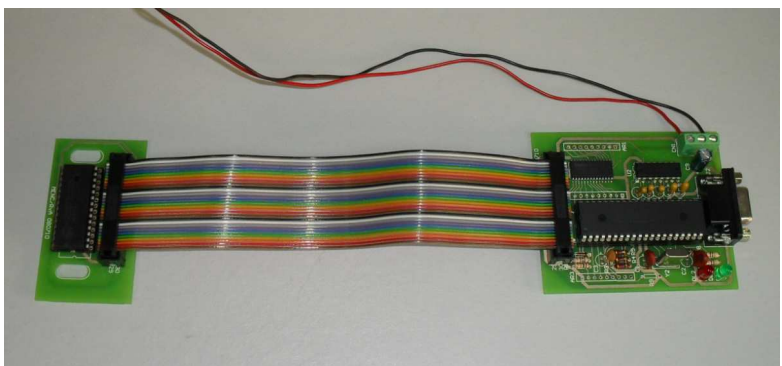

Fig.3. Board for control of the memory and communication with a computer.

-Test set up and data acquisition system. Figure 4 shows a graphic description of laser irradiation system. After decapsulation and mounted on a printed circuit board, the circuit with the memory is mounted on a motorized stage in the three XYZ axes of movement with an accuracy of 0.1 microns in its movement. The laser beam is focused by a microscope objective and long working distance, magnification 50X, suitable for infrared light, achieving a diameter of "spot" on the order of 1 to 1.5 microns for the wavelength used. The laser spot location may be observed with an infrared CCD camera to allow the correct placement of the laser. The simultaneity of experiments in the CLUR imposes a working frequency of the laser at $1 \mathrm{kHz}$. This means that at each point affect a few thousand pulses per second. In principle, the working frequency is a serious problem because to ensure that errors occurring in the memory and that these are correctly accounted needed that impact a single pulse on the memory, and again not influence the next until the memory has been checked, the result is stored and the memory has been displaced to be irradiated in the following point. The time spent in check all the memory and write the result is greater than the time between pulses operating at a frequency of $1 \mathrm{kHz}$, that is $1 \mathrm{~ms}$, so it is necessary to ensure that impact on the memory only one pulse and not affect the next until the memory is ready to receive the next shot, that is, until the above actions have been performed. To achieve it is necessary use of the shutters, through which, and with proper configuration of time so that both are open for $1 \mathrm{~ms}$, ensures that actuate them can only pass a single pulse that will impact on memory and not will actuate again until the memory is ready again. Moreover, by using such shutters is achieved that on each point impact exactly the same radiation flux. Figure 5 shows a real image of the system described. All elements are controlled by a timing routine which is programmed by LabView. This synchronization routine ensures that there is enough time to check the memory, this is, read it and write in a text file all information related to errors (number of errors, direction and error occurred) before the next pulse of energy arrives at the device. Thus, we can perform a complete study of the device, both in two or three dimensions. 


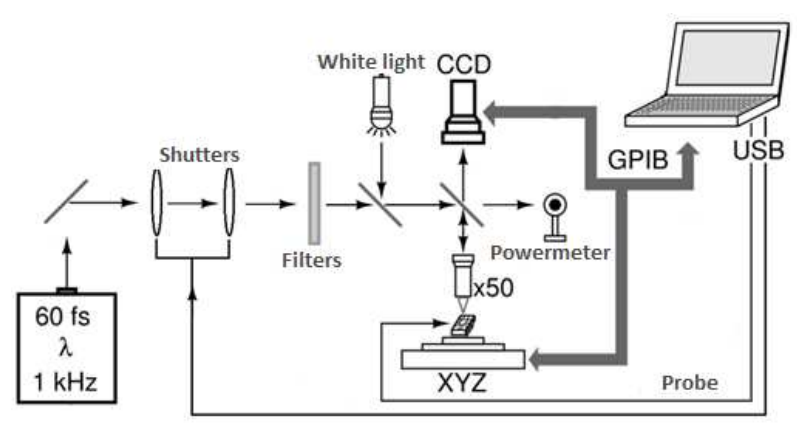

Fig. 4. Schematic of the set-up implemented.

-Laser irradiation. The complete scanning of the whole surface is performed following the state diagram shown in figure 6 . In more detail, by means of the program developed in LabView memories are radiated in the following sequence:

1. Memory is written to the selected pattern.

2. Is positioned the laser focus on a reference point for taking after initial relative positions. For it is used the lay-out of the chip (Fig. 1 and 2) properly calibrated.

3 . From the reference point movement of focus will be in constant steps in the Y direction and once the sweep across that row returns at the beginning of it to forward a step the same as above, but in the direction X. After that the next row is scanned.

4. Radiation and reading errors are automatically performed in the LabView program.

a. It acts on the shutters to achieve a single pulse on the point which is positioned the focus of the beam.

b. It then reads all of the memory cells and compares their state with the initially stored pattern.

c. Transmitted to PC the comparison result and stored it in a file.

d. It reverts to original state the full content of the memory.

e. You move the chip to achieve the next position and starts a new cycle.

5. The results of radiation are analyzed once finished the scanning sequence of all memory and are superimposed on the photograph of the lay-out of the chip.

\section{RESULTS}

During radiation laser process were detected several types of errors such as SEU (Sigle Event Upset), MBU (Multiple Bit Upset) and MCU (Multiple Cell Upset). Note that during the process of determining the radiation energy to CYPRESS memory was observed that for higher energy values, from $170 \mathrm{pJ}$, occurred micro latch-up. Is interesting to note that these micro latch-up does not break the memory, only produced a malfunction recoverable restarting supply voltage.

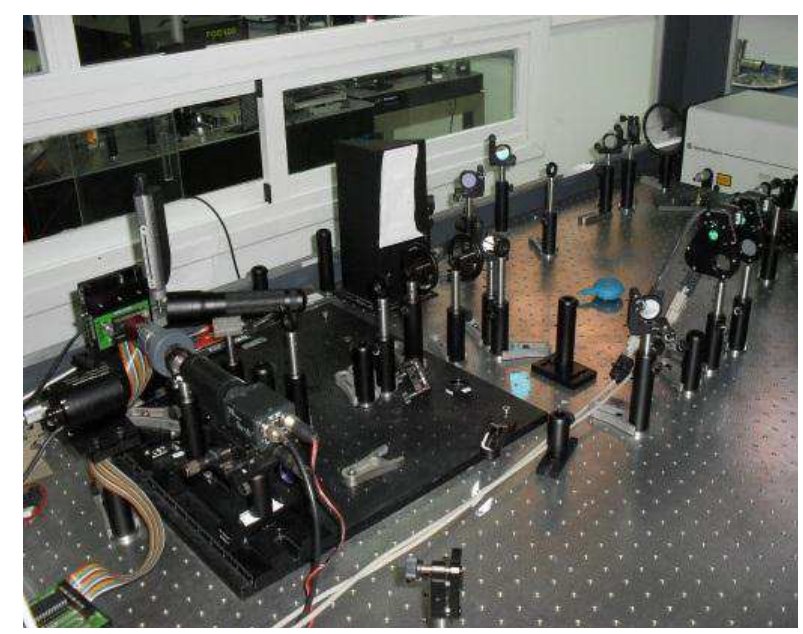

Fig.5. Photography of the set-up implemented at the Centre of Femtosecond Multiphoton Spectroscopy.

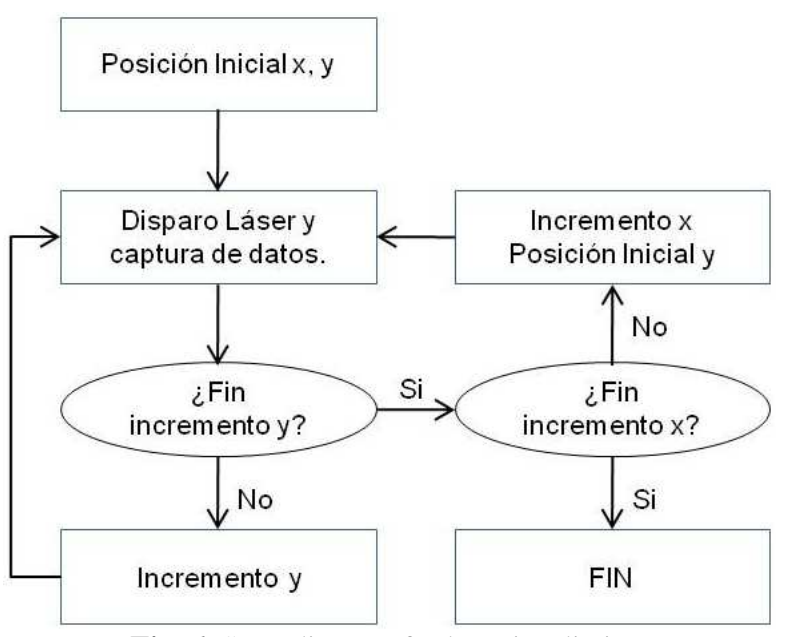

Fig. 6. State diagram for laser irradiation.

From text file result of the radiation we can perform a sensitivity map of the memory, identifying potential error areas and how many errors occur simultaneously.

The sensitivity map for memories ALLIANCE and CYPRESS with a wavelength of $800 \mathrm{~nm}$, an energy of $132 \mathrm{pJ}$ and $154 \mathrm{pJ}$ respectively, and written with the pattern "01010101" are depicted in the following figures (Fig. 7 and 8).

The sensitivity map gives an idea with a simple glance of the sensitivity of the device and we can see which areas are most likely to generate faults.

In both memories is observed two channels or slits without errors that correspond to the two horizontal lines seen in the layout of the chip (Fig. 1 and 2) and probably are metal lines or areas where the logic is implemented. 

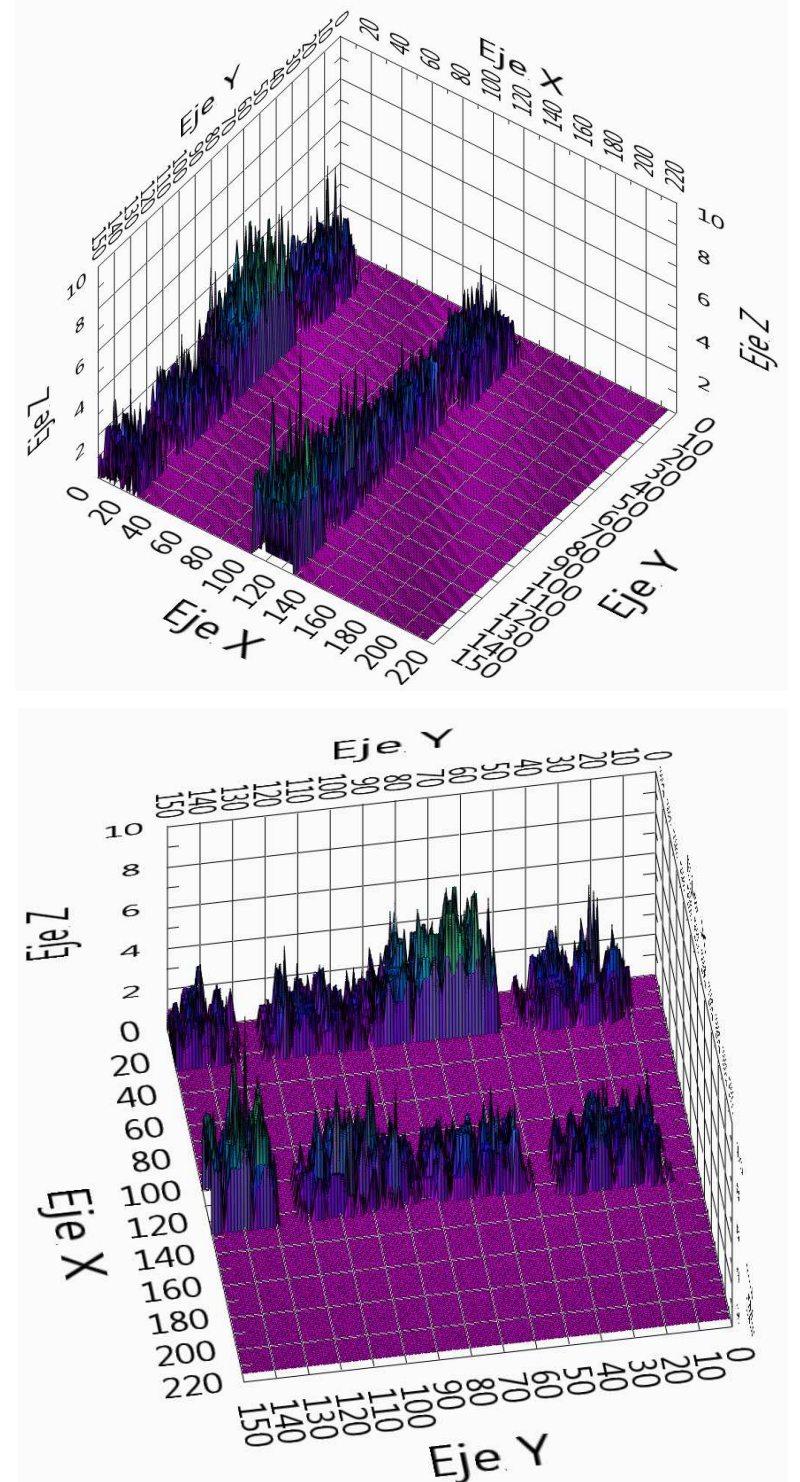

Fig. 7. Sensitivity map for the memory AS6C6264 (Alliance).

Comparing both sensitivity maps is noted that the memory ALLIANCE presents sensitive zones more uniform, whereas in the memory CYPRESS we can see more more variety in sensitivity of the zones, that is, extremely sensitive areas and high, medium an low sensitive areas.

\section{CONCLUSION}

In conclusion, it is demonstrated that the laser can induce errors in SRAM memories, both SEUs as MCUs and even micro-latch.

The laser system of emulation space environment becomes a tool for generating faults on electronic devices of high interest in space electronics technology.
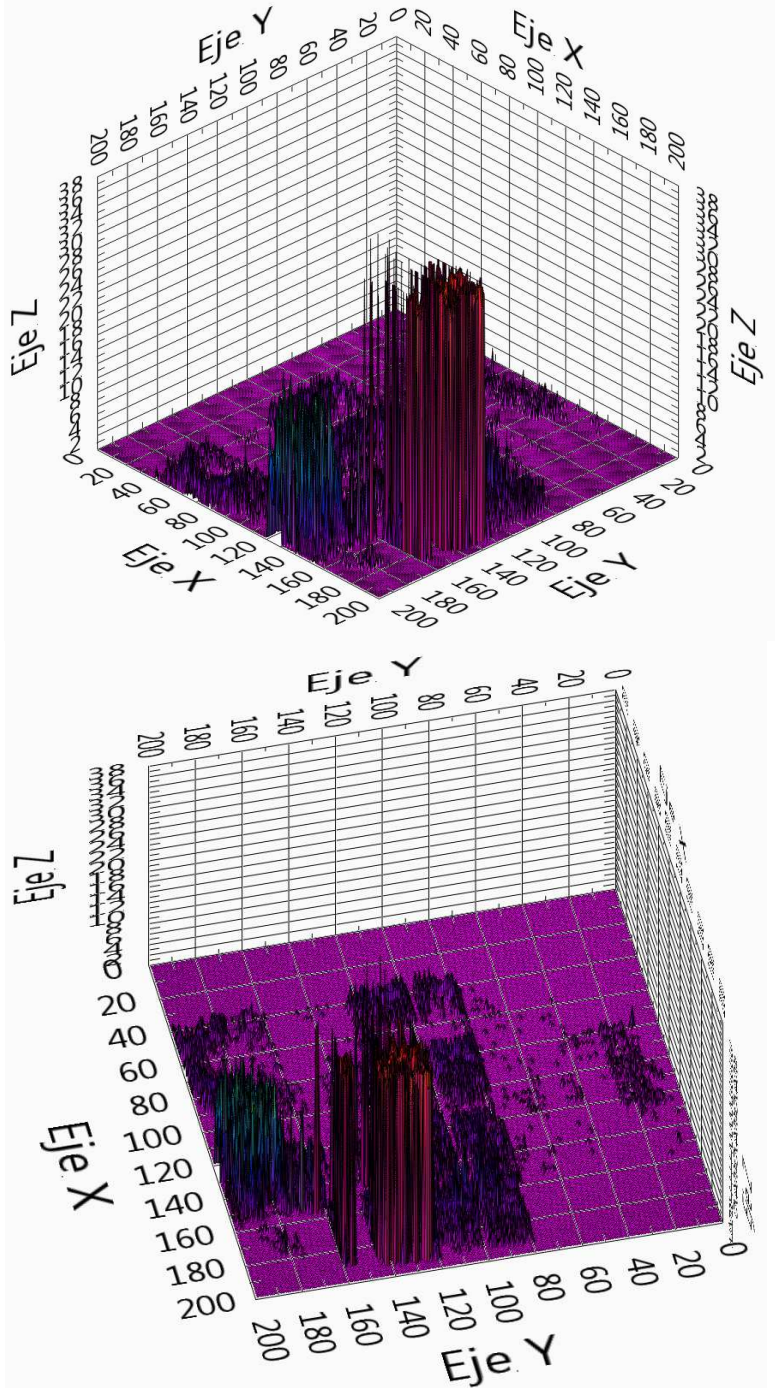

Fig. 8. Sensitivity map for the memory CY62256 (Cypress).

Moreover, counting all errors can be calculated the cross section of the memory. Performing a more exhaustive analysis of the errors caused and its address is possible to know the topology of memory, that is, to know the separation of the cells, interleaving ... So the laser is also a very useful tool in reverse engineering.

\section{References}

[1] I. Lopez-Calle, F. J. Franco, J. G. Izquierdo, \& J. A. Agapito, "LASER System for Space Environment Emulation", IEEE Spanish Conference on Electron Devices, Palma de Mallorca (Spain), pp. 1-4, Feb 2011. [2] F. J. Franco, I. Lopez-Calle, J. G. Izquierdo, and J. A. Agapito, "Modification of the LM124 single event transients by load resistors," IEEE Transactions on Nuclear Science, vol. 57, no. 1, pp. 358-365, Feb. 2010.

[3] F. Miller, "Interest of laser test facility for the assessment of natural radiation environment effects on integrated circuits based systems", 7th RADECS, pp. 199-209, 2003. 